\title{
ESR and Radiocarbon Dating of Gut Strings from Early Plucked Instruments
}

\author{
Sumiko Tsukamoto ${ }^{1, *}$, Taro Takeuchi ${ }^{2}$, Atsushi Tani ${ }^{3}{ }^{(0)}$, Yosuke Miyairi ${ }^{4}$ and \\ Yusuke Yokoyama ${ }^{4}$ \\ 1 Leibniz Institute for Applied Geophysics, Stilleweg 2, 30655 Hannover, Germany \\ 2 The consortium for Guitar Research, Sidney Sussex College, Cambridge CB2 3HU, UK; guitarouk@aol.com \\ 3 Graduate School of Human Development and Environment, Kobe University, Kobe 657-8501, Japan; \\ tani@carp.kobe-u.ac.jp \\ 4 Atmosphere and Ocean Research Institute, The University of Tokyo, Chiba 277-8564, Japan; \\ miyairi@aori.u-tokyo.ac.jp (Y.M.); yokoyama@aori.u-tokyo.ac.jp (Y.Y.) \\ * Correspondence: sumiko.tsukamoto@leibniz-liag.de
}

Received: 14 October 2019; Accepted: 17 January 2020; Published: 28 January 2020

check for updates

\begin{abstract}
Early European plucked instruments have recently experienced a great revival, but a few aspects remain unknown (e.g., the gauge of gut strings). Here we report, for the first time, that the electron spin resonance (ESR) signal intensity of oxidized iron, Fe(III), from gut strings at $g=2$ increases linearly with age within a few hundred years. The signal increase in the remaining old strings on early instruments can be used to judge if they are as old as or younger than the instrument. Obtaining the authenticity information of gut strings contributes to the revival of the old instruments and the music.
\end{abstract}

Keywords: ESR; gut strings; Fe(III); early plucked instruments; radiocarbon

\section{Introduction}

Electron spin resonance (ESR) has been utilized as a geochronometer, based upon the increase in the number of trapped electrons and holes in crystal lattices induced by natural radiation with time [1-3]. ESR also detects unpaired electrons in organic radicals and transition metals in organic substances; the intensity of such ESR signals also increases with time, mainly by thermal activation processes. The possibility of dating organic materials using organic radicals was first tested using potato crisps [4]. The day-by-day increase in the organic radical intensity was used to estimate the production date of the potato crisps. ESR signals of organic radicals and Fe(III) in organic matters, e.g., animal skins, papers, silks and mummies were also investigated, and the intensity showed a positive correlation with age [5-8]. Furthermore, Fe(II) in heme-proteins in human blood starts to oxidize to $\mathrm{Fe}(\mathrm{III})$ after exposure to air, and therefore the increase in Fe(III) in bloodstains detected by ESR has been suggested for use in forensic investigations [9].

In this study, we test the potential for establishing a relative chronology of gut strings from early plucked instruments using the ESR signals of Fe(III) and organic radicals. Gut strings are made from sheep guts containing heme-proteins with Fe(II), which is hardly detected with ESR. Once Fe(II) changes to $\mathrm{Fe}(\mathrm{III})$ by the catalytic cycle reaction of the heme-proteins or oxidation reaction, it becomes detectable with ESR. The $g$-values of the Fe(III) signals in oxidized heme-proteins are affected by the ligand fields [10-12]; for instance, the Fe(III) signal at $g=6$ (high spin state, $S=5 / 2$ ) is originated from methemoglobin coordinated with $\mathrm{H}_{2} \mathrm{O}$, whereas the signal at $g=2$ (low spin state, $S=1 / 2$ ) is that coordinated to $\mathrm{CN}^{-}$or $\mathrm{OH}^{-}[12,13]$. 
Early European plucked string instruments (e.g., lutes, early guitars and harp-lutes) had fallen out of use by the middle of the 19th century, but have been revived with the increased interest in early music in the 20th century. Old gut stings are found occasionally on the instruments or in the cases. We examine five old strings from guitars and harp-lutes, which were made in the 19th century, as well as four strings with known ages. Radiocarbon dating of the strings is also conducted for six strings for a comparison. Although radiocarbon dating has been frequently applied to archaeological music instruments [14,15], little work has been done on recent plucked instruments [16]. To our knowledge, this is the first study of radiocarbon dating on modern (19th-20th centuries) gut strings.

\section{Samples and Results}

The nine gut strings used for the ESR investigation are listed in Table 1 . The approximate ages of four gut string samples are known. Two of them (LHE-14 and -27) were obtained shortly before the first measurement of the samples in April 2015. Another string (LHE-15) was acquired around the year 2000. LHE-16 was provided with a memorandum of the supplier, G. Butler and Sons in London, in 1913 (Figure 1a). Five string samples were collected from four original instruments. Two harp-lutes, which are both assumed to have been made ca. 1815, had remaining old gut strings at the bridge. Two string samples (LHE-17A, -17B) were collected from the 14-string harp-lutes (Figure 1b), and a sample LHE-28 was taken from the 12-string instrument (Figure 1c). A separate string from the 14-string harp-lute (LHE-17C) was used for radiocarbon dating. Sample LHE-19 was found in the original case of a guitar, which was made approximately in ca. 1840 by D\&A Roundhloff in London, and LHE-44 was obtained from a guitar, estimated to have been made between 1850 and 1860. All these original instruments were produced and preserved in the United Kingdom.

Table 1. List of sample strings for ESR.

\begin{tabular}{cccc}
\hline Sample ID & Expected Production Year & Weight $\mathbf{( m g )}$ & Instrument \\
\hline LHE-14 & ca. 2013 & 13.6 & \\
LHE-15 & ca. 2000 & 15.3 & \\
LHE-16 & ca. 1913 & 1.5 & 14-string harp-lute \\
LHE-17A & ca. 1815 & 4.9 & 14-string harp-lute (same as above) \\
LHE-17B & ca. 1815 & 5.6 & a case of a D\&A Roundfloff guitar \\
LHE-19 & ca. 1840 & 1.6 & \\
LHE-27 & ca. 2013 & 24.8 & 12-string harp-lute \\
LHE-28 & ca. 1815 & 26.2 & from a guitar \\
LHE-44 & ca. 1850-1860 & 20.1 & \\
\hline
\end{tabular}

(a)

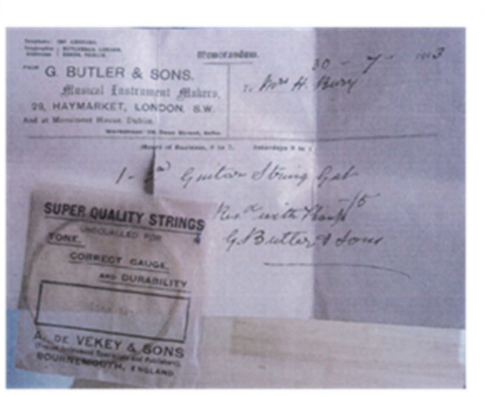

(b)

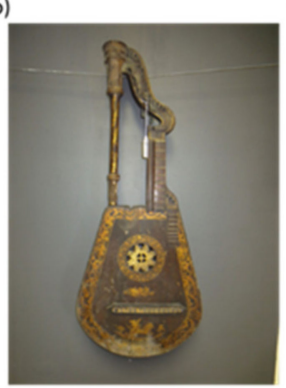

(c)

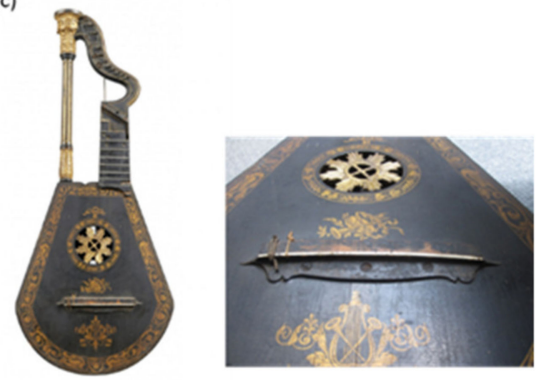

Figure 1. Photos of (a) gut string and its receipt (LHE-16), (b) a 14-string harp-lute (LHE-17A and -17B) and (c) a 12-string harp-lute (LHE-28). Gut string samples were collected from them.

ESR spectra of a recent (LHE-15) and an old string (LHE-17A) are shown for wider $(250 \pm 250 \mathrm{mT}$; Figure $2 \mathrm{a})$ and narrower $(324 \pm 5 \mathrm{mT}$; Figure $2 \mathrm{~b})$ scanned magnetic fields. All gut string samples showed ESR signals from Fe(III) signal at $g=2$ (low spin states, $S=1 / 2$ ) (Figure 2a) and organic radicals 
at around $g=2.005$ (Figure $2 \mathrm{~b}$ ). The Fe(III) signal at $g=6$ (high spin states, $S=5 / 2$ ) (Figure $2 \mathrm{a}$ ) was observed in all samples except LHE-28.
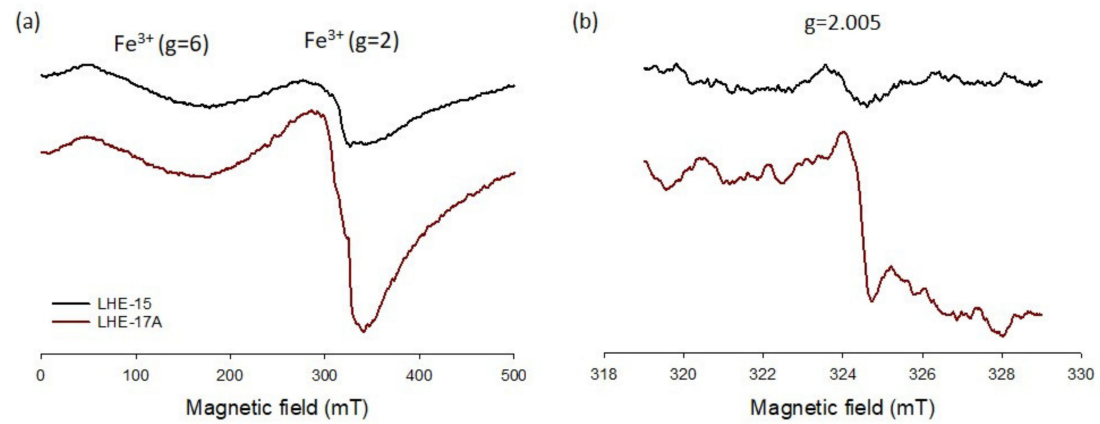

Figure 2. ESR spectra of LHE-15 and -17A for the magnetic field of (a) $250 \pm 250 \mathrm{mT}$ and (b) $324 \pm 5 \mathrm{mT}$. Note that the spectra in $(\mathbf{b})$ were recorded with eight times larger receiver gain than (a) due to the weak signal intensity.

All these strings are assumed to have been produced within $\sim 2$ years before the time of acquisition. Crude but supporting evidence was obtained by the increasing intensity of the Fe(III) signal at $g=2$ within the 11 months for the two modern strings, LHE-14 and LHE-27 (Figure S1). When the intensity is plotted against the time after the first measurement, April 2015, the x-intercept of the fitted straight line points to 15 months and 19 months before the first measurements for LHE-14 and LHE-27, respectively.

The correlation of the ESR signal intensity with age was first examined using four string samples with known ages (LHE-14, -15, -16, and -27). The results are plotted in Figure 3a. Although the number of known age samples is limited, it is clear that the best correlation between the ESR signal intensity and age is obtained from the Fe(III) signal at $g=2$ ( $r=0.95$, Spearman's correlation coefficient). The other two signals showed much less correlation with age, with correlation coefficient of 0.63 for both organic radicals and the $\mathrm{Fe}(\mathrm{III})$ at $g=6$. This indicates that the Fe(III) signal at $g=2$ increases linearly with time, for at least 100 years, and therefore can be used for the relative age estimation of older gut strings.

In Figure $3 b-d$, the three ESR intensities of all samples are plotted against the known string age (filled symbols) or the expected instrument age (open symbols). The results of Fe(III) at $g=6$ and the organic radicals are highly scattered, suggesting these two signals are not suitable as chronometers. For the $\mathrm{Fe}$ (III) at $g=2$, the intensity of three of the five old string samples is consistent with the extrapolated fitted line of the known age strings within the 1- $\sigma$ uncertainty (LHE- 17A, $-17 \mathrm{~B}$, and -28) and one is consistent within the 2- $\sigma$ uncertainty (LHE-19). This suggests that these strings are as old as the instruments. However, the Fe(III) signal $(g=2)$ of LHE-44 has a much lower intensity when compared to the expected intensity from the age of the instrument.

The results of the radiocarbon dating are summarized in Table 2. The method successfully distinguished the strings before and after 1950. The three recent samples (LHE-14, -15, -27) yielded $>100 \%$ modern carbon (pMC), and are therefore judged as modern. The ${ }^{14} \mathrm{C}$ ages of the older three string samples (LHE-16, -17, and -44) ranged from $167 \pm 26$ y BP to $309 \pm 29$ y BP. These were calibrated using OxCal v.3.10 based on Intcal13. Detailed results of the calibrated ${ }^{14} \mathrm{C}$ ages are given in Figure S2. 
(a)

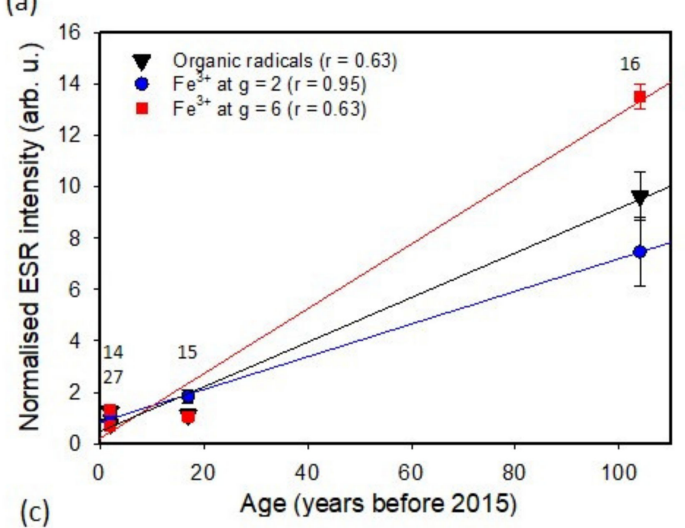

(c)

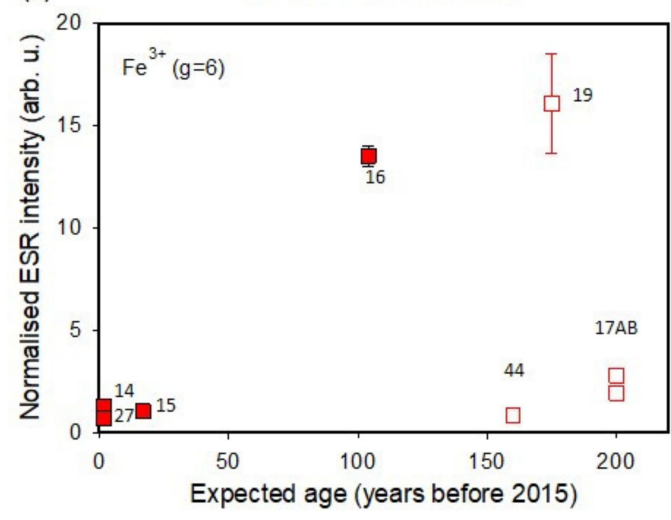

(b)

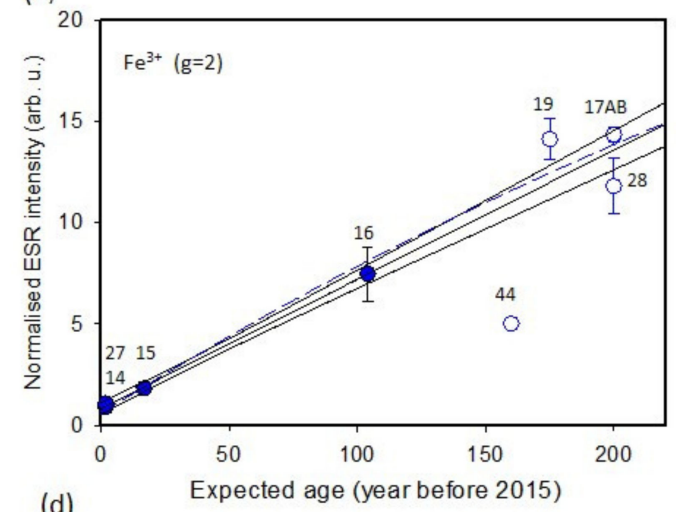

(d)

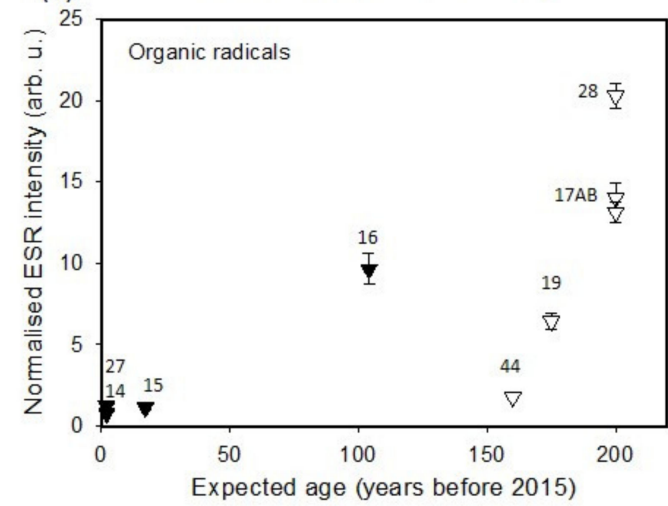

Figure 3. Plot of ESR intensity against the known (filled symbols) or the expected instrument age (open symbols) for (a) all ESR signals of the known age samples, (b) Fe(III) at $g=2$, (c) Fe(III) signal at $g=6$, and (d) organic radicals. The numbers besides the symbols are sample IDs. All error bars in vertical axis are $1-\sigma$ standard error.

Table 2. Results of radiocarbon dating of the strings.

\begin{tabular}{|c|c|c|c|c|c|c|c|}
\hline \multirow{2}{*}{$\begin{array}{c}\text { Lab. Code } \\
\text { YAUT-021827 }\end{array}$} & \multirow{2}{*}{$\begin{array}{c}\text { Sample ID } \\
\text { LHE-14 }\end{array}$} & \multicolumn{2}{|c|}{$\delta^{13} C(\% o)$} & \multicolumn{2}{|c|}{ pMC (\%) } & \multicolumn{2}{|c|}{${ }^{14}$ C Age (Year BP) } \\
\hline & & -25.2 & \pm 1.7 & 104.57 & \pm 0.38 & modern & \\
\hline YAUT-021828 & LHE-15 & -25.4 & \pm 1.8 & 114.34 & \pm 0.42 & modern & \\
\hline YAUT-021830 & LHE-16 & -30.1 & \pm 0.5 & 97.73 & \pm 0.27 & 185 & \pm 22 \\
\hline YAUT-021832 & LHE-17C & -24.0 & \pm 1.7 & 96.23 & \pm 0.35 & 309 & \pm 29 \\
\hline YAUT-021833 & LHE-27 & -16.5 & \pm 1.3 & 118.13 & \pm 0.37 & modern & \\
\hline YAUT-021834 & LHE-44 & -20.4 & \pm 1.3 & 97.95 & \pm 0.32 & 167 & \pm 26 \\
\hline
\end{tabular}

\section{Discussion}

The oxidation of $\mathrm{Fe}(\mathrm{II})$ to $\mathrm{Fe}(\mathrm{III})$ should be sensitive to various environmental factors, e.g., temperature and moisture. As shown in Figure $3 \mathrm{a}$, the very good positive correlation of the Fe(III) signal at $g=2$ is probably due to the fact that all the string samples used in this study were preserved in the United Kingdom, and therefore the environmental factors were similar. A possible explanation of contrasting correlation with age for these two types of Fe(III) is that the low spin heme-proteins are contained relatively uniformly in all gut strings, but the concentration of the high spin heme-proteins is different from sample to sample.

The observed increase in the Fe(III) signal at $g=2$ does not show clear tendency toward saturation, although it is natural to assume that the signal intensity reaches saturation over a longer time period, since a limited number of Fe(II) in heme-proteins should be available in the gut strings. The blue dashed line in Figure $3 b$ shows a regression line, fitted to a single saturation exponential function when all Fe(III) data at $g=2$ except LHE-44 are used. The fitted line is not significantly deviated from the linear regression line (middle of black solid lines in Figure 3b), suggesting that the signal increase is 
still very close to linear for $\sim 200$ years. This result is in contrast with the study of the Fe(III) signal in coagulated human blood; in which the intensity of high spin state Fe(III) reached close to saturation in a few hundred hours at room temperature after coagulation [9], presumably because the Fe(III) in blood was directly exposed to air and the oxidization process was faster.

To investigate how the Fe(III) intensity at $g=2$ in gut strings changes over a longer timescale, aging experiments were conducted using LHE-27 by heating at 60 and $70{ }^{\circ} \mathrm{C}$ in air. The result is shown in Figure S3. At $60^{\circ} \mathrm{C}$, the signal increased only about $14 \%$ from the modern string intensity and reached saturation, whereas at $70{ }^{\circ} \mathrm{C}$ the signal intensity once increased slightly then decreased. In nature, the gut strings of original instruments of $\sim 200$ years old (LHE-17A, $-17 \mathrm{~B},-19$, and -28) showed 12-14 times larger signal intensity than the modern strings. We conclude that the heating at higher temperature cannot reproduce the natural signal growth.

Using the linear increase in the Fe(III) signal $(g=2)$ of gut strings, it is possible to estimate the age ranges of strings with unknown ages. For the five string samples obtained from early plucked string instruments, the consistent ESR signal intensity with the linear regression line of the known age strings for the four samples (LHE-17A, $-17 \mathrm{~B},-19$, and -28) indicate that the strings are as old as the instruments. This indicates that these instruments (early guitars and harp lutes) were not used for a long time after they were made. This also suggests that remaining strings can be used as a record of the original gauges. One string sample, LHE-44 yielded much a lower Fe(III) intensity than expected considering the instrument age. By comparing the intensity with the regression line, it is assumed that this string was made 60-70 years before 2015 .

Currently, it is still unknown how variable the signal increase is in different storage conditions. More data of string samples with known ages and from different environments should be accumulated to establish the method to be a robust dating technique.

AMS radiocarbon dating can accurately judge whether a gut string was made before or after 1950 (Table 2). However, calibration of a ${ }^{14} \mathrm{C}$ age into a calendar age generates a large uncertainty (Figure S2). Sample LHE-16, which was sold in 1913, yielded a calibrated age of AD1660-1690 (18.2\%), AD1730-1810 (53.4\%) and later than AD1920 (23.8\%) in 95.4\% probability. As mentioned above, the ESR of LHE-44 gave an assumption that the string was much younger than the instrument (AD1850-1860). However, the calibrated ${ }^{14} \mathrm{C}$ age of this sample yielded AD1660-1700 (16.7\%), AD1720-1820 (51.9\%), AD1830-1880 (6.5\%) and later than AD1910 (20.5\%), which cover both the instrument age and ESR age of this string. For LHE-17, the calibrated ${ }^{14} \mathrm{C}$ age yielded a much older age than the instrument (ca. 1815) - AD1480-1650 - which might be due to the contamination of old carbon in the production process.

\section{Methods}

All ESR measurements were conducted using a JEOL-FA-100 X-band spectrometer at the Leibniz Institute for Applied Geophysics, Hannover, Germany. The string samples, which were cut into $\sim 1 \mathrm{~cm}$ length, were inserted into quartz glass tubes of $4 \mathrm{~mm}$ outer diameter ( $3 \mathrm{~mm}$ inner diameter), and used for the ESR measurements. The measurements were made at room temperature, three times in April 2015, January 2016 and February 2016. The measurement parameters for the Fe(III) signals were; $250 \pm 250 \mathrm{mT}$ magnetic field, $1 \mathrm{~mW}$ microwave power, 1 min scan time for 3 times, $0.5 \mathrm{mT}$ modulation amplitude and $0.1 \mathrm{~s}$ time constant. The organic radical signal $(g=2.005)$ was measured with the following conditions; $324 \pm 5 \mathrm{mT}$ magnetic field, $2 \mathrm{~mW}$ microwave power, $30 \mathrm{~s}$ scan time up to 50 times, $0.3 \mathrm{mT}$ modulation width and $0.1 \mathrm{~s}$ time constant. The mean peak to peak ESR intensity of the three measurements and its $1-\sigma$ standard error was calculated. For one sample, which we obtained in December 2015 (LHE-44) the mean signal intensity of two measurements was used. Since the available sample mount varied significantly (1.5 and $26 \mathrm{mg}$; Table 1), all ESR signal intensities were normalized to the weight of each string sample.

The radiocarbon dating of the six string samples (LHE-14, -15, -16, -17C, -19 and -44 ) was conducted with an NEC $250 \mathrm{kV}$ single-stage accelerator mass spectrometer (AMS) at the Atmosphere and Ocean 
Research Institute, The University of Tokyo [17]. The string samples were washed with ultra-pure water and dried. These samples were oxidized using a Vario Micro Cube elemental analyzer manufactured by Elementar Analysensysteme GmbH., and an AMS analytical target was prepared using an automated graphitization process device manufactured by Koshin Rikagaku Seisakusho Co., Ltd (Tokyo, Japan).

\section{Conclusions}

A very good correlation between the Fe(III) signal and the known ages of gut strings was observed. This probably indicates that $\mathrm{Fe}$ (II) in the gutstrings has been oxidized to $\mathrm{Fe}(\mathrm{III})$ with age. We conclude that it is possible to assume whether a gut string is as old as the instrument or much younger, using the correlation of the Fe(III) signal $(g=2)$ with age. Using ${ }^{14} \mathrm{C}$ dating it is also possible to judge a string is a recent one or older, but a calibrated age has a large uncertainty.

Supplementary Materials: The following are available online at http://www.mdpi.com/2409-9279/3/1/13/s1, Figure S1: The increase of the Fe(III) $(g=2)$ signal after the first ESR measurement, April 2015 with time. The production time before the first measurement was roughly estimated by linearly extrapolating the fitted line to zero intensity. Data were normalised to the same factor as was used for Figure 3. Unfortunately further measurements were not achieved due to a problem with the ESR spectrometer, Figure S2: Results of calibration for the radiocarbon ages, Figure S3: Result of the aging experiment of a string sample LHE-27 heated at $60{ }^{\circ} \mathrm{C}$ and $70{ }^{\circ} \mathrm{C}$. The intensity of $\mathrm{Fe}(\mathrm{III})$ at $g=2$ after different durations of heating is shown.

Author Contributions: S.T. and T.T. designed the study. S.T. conducted all ESR measurements and wrote the paper. T.T. collected gut string samples and contributed in the sample descriptions. A.T. contributed to the discussion of the physics of $\mathrm{Fe}^{3+}$. Y.M. and Y.Y. conducted radiocarbon dating and contributed to the corresponding discussion. All authors have read and agreed to the published version of the manuscript.

Acknowledgments: We are very grateful to James Westbrook for providing string samples and photos. Gwynyn Buchanan is thanked for the language edits.

Conflicts of Interest: The authors declare no conflict of interest.

\section{References}

1. Ikeya, M. New Applications of Electron Spin Resonance Dating, Dosimetry and Microspcopy; World Scientific: Singapore, 1993; p. 520.

2. Rink, W.J. Electron spin resonance (ESR) dating and ESR applications in quaternary science and archaeometry. Radiat. Meas. 1997, 27, 975-1025. [CrossRef]

3. Schellmann, G.; Beerten, K.; Radtke, U. Electron spin resonance (ESR) dating of Quaternary materials. Quat. Sci. Jour. 2008, 57, 150-178.

4. Ikeya, M.; Miki, T. A new dating using digital ESR method. Naturwissenschaften 1980, 67, 191-192. [CrossRef]

5. Ikeya, M.; Miki, T. ESR dating of organic materials: from potato-chips to a dead body. Nucl. Tracks. Radiat. Meas. 1985, 10, 909-912. [CrossRef]

6. Miki, T.; Yahagi, T.; Ikeya, M.; Sugawara, N.; Furuno, J. ESR dating of organic substances: corpse for forensic medicine. In ESR Dating and Dosimetry; Ikeya, M., Miki, T., Eds.; Ionics: Tokyo, Japan, 1985; pp. 447-451.

7. Miki, T.; Kai, A.; Ikeya, M. ESR dating utilizing in valency or ligand of transition metals. Jpn. J. Appl. Phys. 1987, 972-973. [CrossRef]

8. Miki, T.; Kai, A.; Ikeya, M. ESR dating of organic substances utilizing paramagnetic degradation products. Nucl. Tracks. Radiat. Meas. 1988, 14, 253-258. [CrossRef]

9. Miki, T.; Kai, A.; Ikeya, M. Electron spin resonance of bloodstains and its application to time after bleeding. Forensic Sci. Intern. 1987, 35, 149-158. [CrossRef]

10. Banerjee, R.; Stetzkowski, F. Heme transfer from haemoglobin and ferrihemoglobin to some new ligands and its implication in the mechanism of oxidation of ferrohemoglobin by air. Biochem. Biophys. Acta. 1970, 221, 636-639. [PubMed]

11. Peisach, J.; Blumberg, W.E.; Wittenberg, B.A.; Wittenberg, J.B.; Kampa, L. Hemoglobin a: an electron paramagnetic resonance study of the effects of interchain contacts on the heme symmetry of high-spin and low-spin derivatives of ferric alpha chains. Proc. Nat. Acad. Sci. USA 1969, 63, 934-939. [CrossRef] [PubMed]

12. Hori, H. Analysis of the principal g-tensors in single crystal of ferrimyoglobin cpmplexes. Biochem. Biophys. Acta 1971, 251, 227-235. [PubMed] 
13. Masuda, H.; Tagam, T. The crystal and molecular structures of metalloporphyrin complexes with anomalous electronic states. Jour. Crystal. Soc. Jpn. 1984, 25, 289-298. [CrossRef]

14. Zhang, J.; Harbottle, G.; Wang, C.; Kong, Z. Oldest playable musical instruments found at Jiahu early Neolithic site in China. Nature 1999, 401, 366-368. [CrossRef] [PubMed]

15. Conrad, N.J.; Malina, M.; Münzel, S.C. New flutes document the earliest musical tradition in sourthwestern Germany. Nature 2009, 460, 737-740. [CrossRef] [PubMed]

16. Durier, M.G.; Bruguière, P.; Hatté, C.; Vaiedelich, S. Radiocarbon dating of legacy music instrument collections: Example of traditional indian Vina from the Musée De La Musique, Paris. Radiocarbon 2018, 61, 17-22. [CrossRef]

17. Yokoyama, Y.; Miyairi, Y.; Aze, T.; Yamane, M.; Sawada, C.; Ando, Y.; de Natris, M.; Hirabayashi, S.; Ishiwa, T.; Sato, N.; et al. A single stage Accelerator Mass Spectrometry at the Atmosphere and Ocean Research Institute, The University of Tokyo. Nucl. Inst. Meth. Phys. Res. B 2019, 455, 311-316. [CrossRef]

(C) 2020 by the authors. Licensee MDPI, Basel, Switzerland. This article is an open access article distributed under the terms and conditions of the Creative Commons Attribution (CC BY) license (http://creativecommons.org/licenses/by/4.0/). 